Davis, G. H. G. \& Hayward, A. C. (1955). J. gen. Microbiol. 13, 533-540

\title{
The Stability of Lactobacillus Strains
}

\author{
By G. H. G. DAVIS ANd A. C. HAYWARD \\ Department of Bacteriology, University of Birmingham
}

\begin{abstract}
SUMMARY : Reported changes of pure strains of Lactobacillus from the heterofermentative to the homofermentative type, with concomitant changes in many characters of recognized taxonomic importance, were investigated. No evidence was obtained that such gross variations occur. Inadequate initial purification and subsequent contamination between strains of different physiological types, were found to produce effects which could be mistaken for such changes.
\end{abstract}

The literature contains many allusions to the morphological and physiological variability of Lactobacillus in artificial culture. Most of these reports concern minor variations in, for instance, fermentation reactions (Rosebury, Linton \& Buchbinder, 1929; McIntosh, James \& Lazarus-Barlow, 1922, 1924). In recent years such variations have been recognized as of little taxonomic importance and do not impede the accurate classification of lactobacilli.

Although Cannon (1924) stated that no appreciable variation in fermentation reaction powers occurred as a result of prolonged culture of lactobacilli, Howitt (1930) regarded prolonged culture as a necessary stabilizing process, without which reliable classification could not be attained. Harrison $(1942 a, b)$ reported a type of variation in the physiological characters of oral Lactobacillus strains during the course of prolonged artificial culture which appeared to support Howitt's theory and was clearly of major taxonomic importance. Harrison (1942 $a$ ) classified oral lactobacilli into two main types, groups A and $B$, on the basis of mannitol and sorbitol fermentation and acid production in milk. In the light of modern studies it is almost certain that group A represented the homofermentative $L$. case $i$ and group B, the heterofermentative L. fermenti (Davis, 1955). Harrison (1942b) claimed that group B strains (mannitol- and sorbitol-negative) tended to acquire the morphological, physiological and serological characteristics of group A when maintained for some time in artificial culture. Although this apparent change from the group B to the group A type was observed in many strains, Harrison only found one group A strain which changed to the group B type. Possible explanations of these observations offered by Harrison were the acquisition of protoplasmic characters, development of adaptive enzymes, selection of variant cells or colonies, or the initial presence of mixed cell populations in apparently pure cultures.

Harrison's work was amplified by Coolidge, Williams, Ebisch \& Hodges (1949), Coolidge (1950), Hodges, Coolidge \& Harrison (1951), and Coolidge (1951) who claimed that the changes observed in physiology, serology, morphology and nutrition, in certain strains, were in fact the result of changes from the heterofermentative to the homofermentative type. The disappearance 
of the characteristically heterofermentative requirement for thiamin, and changes in the end products of glucose fermentation were recorded for heterofermentative cultures, derived from single cell isolates, in which metabolic changes were considered to have occurred. Nutritional adaptation during growth upon thiamin-free media was not found to produce this change; it appeared to occur very readily, however, in strains subjected to washing in $0.85 \%$ saline solution. Washing in other metallic salt solutions did not appear to affect the rate of change. These studies gave the impression that the gross metabolic changes reported were caused by some process resembling a genetic mutation of the entire cell population in the cultures examined. In view of the taxonomic implications of such a process, if it should indeed prove to occur, the present investigations were made.

\section{METHODS}

The strains employed were of oral origin, maintained and classified by the methods previously described (Davis, 1955; Davis, Bisset \& Hale, 1955). The techniques used were essentially the same as those of Coolidge and co-workers, but it should be noted that the medium employed, tomato juice (TJ) broth and agar, and the methods of strain characterization were somewhat improved as a result of recent studies in this field (see Davis, 1955, for literature). In all, 7 strains of Lactobacillus casei and 9 of L. fermenti were employed in the following experiments as homofermentative and heterofermentative representatives of the genus respectively.

\section{EXPERIMENTAL}

Experiment 1. Effect of washing cells in $0.85 \%$ sodium chloride. Three strains of Lactobacillus casei and two of $L$. fermenti were checked for purity by repeating three times the selection of discrete colonies from $48 \mathrm{hr}$. surface cultures upon TJ agar. These were then inoculated from $24 \mathrm{hr}$. TJ broth cultures using capillary pipettes, to gas production and fermentation reaction media (Davis, 1955) to check the strain characteristics, and into duplicate TJ broths which were incubated for $24 \mathrm{hr}$. at $37^{\circ}$. One broth culture was then used to inoculate fermentation tests and $\mathrm{TJ}$ broth direct; the other was washed three times in $\mathbf{0 . 8 5} \%$ sodium chloride solution and used to inoculate fermentation tests and $\mathrm{TJ}$ broth. The total period of time for which the cells of any one subculture were in contact with saline solution was never more than 50 min., during washing and resuspension. This was repeated every $48 \mathrm{hr}$. for 20 days, giving two parallel series of cultures for each strain, one washed in saline between subcultures and the other acting as an unwashed control series. The strain characters, as shown by fermentation reaction patterns, were recorded for each subculture of the washed and control series.

Results. The original fermentation patterns (subculture no. 1) of the five strains are shown in Table 1 . During the course of ten successive transfers in TJ broth without washing in saline, no variation in the fermentation patterns of homofermentative strains $(81,5,99)$ occurred. Of the heterofermentative 
strains, no. 68 fermented mannitol weakly (colour change required 12 days to become apparent) in the 6th subculture but not in subsequent ones. Strain 119 fermented salicin in the 3rd and 4th subcultures only. At the end of the experiment the fermentation patterns of all strains were exactly the same as at the beginning.

\section{Table 1. Original fermentation patterns}

$\begin{array}{rccccccccc}\text { Strain Raffinose } & \text { Xylose } & \text { Lactose } & \text { Arabinose } & \text { Salicin } & \text { Rhamnose } & \text { Mannitol } & \text { Sorbitol } & \text { Species } \\ \mathbf{8 1} & - & - & + & - & + & - & + & + & \text { L. casei } \\ \mathbf{5} & - & - & + & - & + & + & + & + & \text { L. casei } \\ \mathbf{9 9} & - & - & + & - & + & - & + & - & \text { L. casei } \\ \mathbf{6 8} & + & - & + & - & - & - & - & - & \text { L. fermenti } \\ \mathbf{1 1 9} & + & - & + & - & - & - & - & - & \text { L. fermenti }\end{array}$

All fermentations apparent within 6 days.

In the series which underwent saline washing, homofermentative strains showed no variation from the original fermentation patterns. Strain 68 fermented sorbitol and mannitol weakly in the first suspension, but subsequent suspensions were inactive toward these sugars. Strain 119, however, showed definite and permanent changes in its fermentation patterns as the experiment proceeded. From the first saline suspension, mannitol and sorbitol were fermented; from the second, salicin, and from the ninth, rhamnose were also fermented. The only sugars not fermented by the tenth suspension were therefore xylose and arabinose. The cumulative effect was indicated by an ability to ferment greater numbers of the sugars tested, and an increased rate of fermentations in later suspensions. For instance, the second suspension required 14 days to ferment sorbitol, whereas the tenth suspension fermented sorbitol in only 4 days.

Superficially this result resembles those of Harrison and his co-workers. The fermentation of mannitol and sorbitol by strains originally incapable of fermenting these sugars was the main criterion of change used by Harrison $(1942 a, b)$. The occurrence of a marked and permanent change in a heterofermentative strain only, and that only when washed in saline, also appeared to confirm the claims of Coolidge. However, when at the end of the experiment, the final cultures of strain 119, derived from both the washed and control series were again tested for gas production, both were positive. The washed culture was thus shown to have remained heterofermentative in character. It was also observed that although the washed culture had acquired the fermentation characters of Lactobacillus casei, it had not lost those of L. fermenti. These facts suggested that the washed culture contained lactobacilli of both the $L$. casei and $L$. fermenti type. This was checked by culturing the final washed and unwashed cultures of strain 119 upon TJ agar, selecting six representative colonies from each and examining their characteristics. The results are shown in Table 2.

The ready separation of the two components ( 1 and 2 ) of the washed culture, showed conclusively that the aberrant fermentation reactions recorded during the course of the experiment were caused by the occurrence of a mixed cell 
population containing lactobacilli of both the Lactobacillus casei and L. fermenti type.

This experiment was repeated using 3 strains of Lactobacillus casei and 5 of $L$. fermenti not examined in the first experiment. 4 of the L. fermenti strains were found to contain cells of the $L$. casei type after only one subculture and washing in saline. Unwashed subcultures remained pure through ten transfers in TJ broth.

\begin{tabular}{|c|c|c|c|c|}
\hline \multicolumn{5}{|c|}{ Table 2} \\
\hline Strain 119 & Colony & Cell form & Gas & Fermentations \\
\hline Unwashed & Large, semi-rough & $\begin{array}{l}\text { Single and paired } \\
\text { short bacilli }\end{array}$ & + & Raffinose and lactose \\
\hline \multirow[t]{2}{*}{ Washed } & (1) Large semi-rough & $\begin{array}{l}\text { Single and paired } \\
\text { short bacilli }\end{array}$ & + & Raffinose and lactose \\
\hline & (2) Small smooth & Bacilli in chains & - & $\begin{array}{l}\text { Lactose, rhamnose, } \\
\text { salicin, mannitol } \\
\text { and sorbitol }\end{array}$ \\
\hline
\end{tabular}

It is clear that the most probable explanation of these results was simple contamination of Lactobacillus fermenti cultures by the $L$. casei cultures handled in the same experiment. The accurate designation of strains to certain species now possible in this genus, makes the explanation of the mixed cultures, as being due to contamination, much more easily discernible than it is if the strains are only known as heterofermentative or homofermentative, which was in fact the only designation that Coolidge and his colleagues could give to their strains. The experiments of Coolidge involved the handling of homofermentative and heterofermentative strains at the same time, as in Expt. 1 above, and contamination could therefore have occurred. It is certain that the process of centrifuging, decanting and resuspending cultures, although conducted with normal sterile precautions, is more open to contamination than is that of straightforward subculturing. Coolidge et al. (1949) state: 'The coincidence of changes in the two organisms (two heterofermentative strains) on the same day after repeated failure to change, suggests an uncontrolled factor in the medium, technique or environment'; and later, Hodges et al. (1951) write: 'Some factor associated with the preparation of washed cell suspensions of the organisms increases the incidence of the change'. In our opinion, contamination was the unknown factor.

Experiment 2. At different times and by different operators, 2 strains of Lactobacillus fermenti were separately treated as in Expt. 1. Both strains were from stock cultures of which previous subcultures had become mixed when used in Expt. 1. They were examined alone, i.e. no homofermentative or other heterofermentative strains were handled at the same time.

Result. In neither case did any change occur in the fermentation patterns of washed or unwashed subcultures, and at the end of the experiments both strains were pure and had their original characters. This showed that in the absence of possible contaminants, the strains were not affected by the treatment administered to them, and was considered to give support to the present contamination theory. It is possible that the mixed cell populations detected in 
Expt. 1 might arise from the initial presence, in apparently pure cultures, of undetected minorities of alien cells which, under the action of selective forces, increased to detectable proportions.

Experiment 3. The question of initial impurity was satisfactorily answered by using cultures derived from single cells isolated with the aid of the De Fonbrune micromanipulator. One strain of Lactobacillus casei and one of L. fermenti were thus purified and examined together, as in Expt. 1.

Result. In the series of cultures subjected to washing, mixing of the two strains occurred quite rapidly, the presence of cells of the Lactobacillus casei type in the cultures of $L$. fermenti was the most easy to detect, but contamination of the $L$. case $i$ cultures by cells of the $L$. fermenti type was also found to occur. No changes occurred in the unwashed control cultures.

Subsidiary experiments were also made to determine the possible selective action of sodium chloride at various concentrations and over various periods of time upon Lactobacillus strains of the two species being tested. The viable count method of Miles \& Misra (1938) was used, as well as qualitative tests by cultural methods to determine their survival in, and tolerance of sodium chloride. The results showed that no great distinction existed between L. case i and L. fermenti in this respect, and it was therefore unlikely to be a factor in the selection of cells of either type in initially impure cultures. It is certain, however, that in most media used for Lactobacillus cultivation, cells of the casei type grow more rapidly and luxuriantly at $37^{\circ}$, than do those of other species. In consequence an initial, undetectable minority of such cells may increase in proportion in succeeding subcultures and even completely overgrow the culture. This is undoubtedly the manner in which the 'stabilizing effect' of prolonged culture (Howitt, 1930) was produced. The contemporary use of media more adequately designed to meet the nutritional requirements of as many different Lactobacillus types as possible (e.g. Cox \& Briggs, 1954) considerably reduces the chance of such selective action by the medium.

Experiment 4. To examine more closely the occurrence of cross-contamination between the different lactobacilli used in experiments of the type described in Expt. 1, tests were made using a strain of Lactobacillus casei and one of L. fermenti whose colony forms upon TJ agar were clearly recognizable. After each subculture and washing, the control and washed cultures were plated upon TJ agar and checked after growth for $48 \mathrm{hr}$. at $37^{\circ}$ for the presence of foreign colonies. Such colonies were isolated and classified.

Results. Contamination between the two strains was readily detected in the washed series, and each contaminated the other. No contamination occurred in the control unwashed series during ten subcultures.

Experiment 5. One strain of Lactobacillus casei and one of L. fermenti, each derived from single cell isolates were subcultured in four parallel series in TJ broth at $48 \mathrm{hr}$. intervals. One series was an unwashed control, one was triple washed and resuspended in $\mathbf{0 . 8 5} \%$ saline solution, one was similarly treated with $0.05 \%$ manganous chloride solution, and one with quarter strength Ringer's solution between each subculture. Manganous chloride was chosen as a possible agent of genetical mutation, and Ringer's solution as a relatively 
non-toxic suspending fluid. In all, 24 successive subcultures and washings were made for both strains in each series.

Result. From observations of colony forms as in Expt. 4 and by their subsequent classification, contamination was detected in all three series of washed cultures after less than ten transfers. The unwashed series was still pure at the 10th subculture, but contamination did finally occur at the 23rd subculture.

Although Coolidge et al. (1949) reported no changes in cultures washed in solutions other than $\mathbf{0 . 8 5} \%$ sodium chloride, we consider that the actual fluid employed is of little significance in the initiation of 'changes of character'. The important factor is the exposure to contamination by excessive manipulation of the cultures examined.

\section{DISCUSSION}

The 'stabilizing effects' of prolonged artificial culture upon Lactobacillus strains reported by Howitt (1930) and by Harrison (1942) were almost certainly due merely to the gradual purification during maintenance, of strains which were initially impure. Harrison's (1942) observations of changes were similarly the result of the use of mixed cultures, a fact which Harrison realized was possible, but which was not investigated.

In Coolidge's work, the use of single cell isolates precludes the possibility of initial impurity (see Expt. 3, above), but the use of both homofermentative and heterofermentative strains in the experiments, and contamination during the subculturing and washing of cultures is considered by the present authors to be the true explanation of the apparent metabolic changes observed. That these workers did not detect the mixed nature of the supposedly changed cultures was probably because of the complete overgrowth of the contaminant strain and loss of the original culture type, or the lack of techniques suitable for the adequate diagnosis of strains, or both. It also appears certain that the process of centrifuging and washing cultures, although conducted with normal bacteriological precautions, facilitates contamination; the actual solution used for the washing having little or no affect upon the result. Another possible factor assisting contamination in the present study was the use of screw-capped bottles. In these, the carbon-dioxide generated by heterofermentative strains causes a distinct effervescence when shaken rapidly and opened and the bacteria may be disseminated by atomization.

In the taxonomy of Lactobacillus, the acceptance of morphological variability as a characteristic of the genus has undoubtedly led to the description of variation in cultures which were, in fact, simply impure. In consequence, such studies have produced apparent anomalies which only serve to confuse the systematics of the group. Laxminarayana et al. (1952) reported heterofermentative strains of $L$. plantarum which, in our opinion, were no more than cultures containing lactobacilli of both the $L$. casei and $L$. fermenti type. Mixtures of this type have been encountered in this laboratory, and their combined characters can be mistakenly interpreted as those of gas producing strains of $L$. plantarum. In our experience all such cultures have subsequently been resolved into two pure cultures by normal purification techniques. 
So far as we know, no other investigations of the phenomena reported by Coolidge et al. (1949) have been attempted. The interpretation of our own results as due to contamination naturally leads us to believe that the original reports are similarly explicable. The possibility of a genetical change, especially one incurred by the action of saline solution, appears most doubtful, but cannot be entirely ignored. Little or nothing is known of the genetics of Lactobacillus, and because of the extreme importance of such variations, if in fact they occur, some introductory experiments were made to determine whether genetically 'marked' strains of heterofermentative lactobacilli could be persuaded, either by mutation or back-crossing to produce homofermentative variants. These were uniformly negative and will not be described here in detail.

Our findings agree with those of Rogosa et al. (1953) that major variations in taxonomic characters, such as a change from the heterofermentative to the homofermentative type which would imply the instability of the main subgeneric division of the genus (Davis, 1955), do not normally occur. We wish to emphasize that for the purposes of primary purification of lactobacilli, the morphological characters of colonies and cells may be reliably employed as indicators of strain uniformity, as well as inspecies diagnosis.

\section{REFERENCES}

Cannon, P. R. (1924). A biological study of aciduric bacteria. J. infect. Dis. 34, 227.

Coolidge, T. B. (1950). Metabolism of oral lactobacilli. J. dent. Res. 29, 659.

Coolidge, T. B. (1951). Heterofermentative to homofermentative change in lactobacilli. $J$. infect. Dis. 88, 241.

Coolidge, T. B., Williams, N. B., Ebisch, A. E. I. \& Hodges, E. A. (1949). Metabolic changes in oral lactobacilli. J. infect. Dis. 85, 126.

Cox, C. P. \& Briggs, M. (1954). Experiments on growth media for lactobacilli. J. appl. Bact. 17, 18.

Davis, G. H. G. (1955). The classification of lactobacilli from the human mouth. J. gen. Microbiol. 13, 481 .

Davis, G. H. G., Bisset, K. A. \& Hale, C. M. F. (1955). Correlation between morphological and physiological characters in the classification of members of the genus Lactobacillus. J. gen. Microbiol. 13, 68.

Harrison, R. W. (1942a). Studies on lactobacilli. III. Relationship of immunological susceptibility and fermentative capacity. J. infect. Dis. 70, 69.

Harrison, R. W. (1942b). Studies on lactobacilli. IV. Changes in immunological specificity associated with changes in fermentation reactions. J. infect. Dis. 70, 77.

Hodges, E. A., Coolidge, T. B. \& Harrison, R. W. (1951). Further observations on metabolic changes in oral lactobacilli. J. infect. Dis. 88, 237.

HowrTt, B. (1930). Cultural and serological reactions of lactobacilli from the mouth. J. infect. Dis. 46, 351.

Laxminarayana, H., Nambudripad, V. K. N., Lakshminarasim, V., Anantaramiah, S. N., Screenivasamurthy, V. \& Iya, K. K. (1952). Studies on Dahi, 3. Taxonomy of the lactic acid bacteria of Dahi. Indian J. vet. Sci. 22, 27.

McIntosh, J., James, W. W. \& Lazarus-Barlow, P. (1922). An investigation into the aetiology of dental caries. 1 . The nature of the destructive agents and the production of artificial caries. Brit. J. exp. Path. 3, 138. 
McIntosh, J., James, W. W. \& Lazarus-Barlow, P. (1924). An investigation into the aetiology of dental caries. 2. The biological characteristics and distribution of $B$. acidophilus-odontolyticus; 3. Further experiments on the production of artificial caries. Brit. J. exp. Path. 5, 175.

Mrles, A. A. \& Misra, S. S. (1938). The estimation of the bactericidal power of the blood. J. Hyg., Camb. 38, 732.

Rogosa, M., Wiseman, R. F., Mitchell, J. A., Disraely, M. N. \& Beaman, A. J. (1953). Species differentiation of oral lactobacilli from man including descriptions of Lactobacillus salivarius nov.spec. and Lactobacillus cellobiosus nov.spec. J. Bact. 65, 681.

Rosebury, T., Linton, R. W. \& Buchbinder, L. (1929). A comparative study of dental aciduric organisms and Lactobacillus acidophilus. J. Bact. 18, 395.

(Received 27 June 1955) 